\title{
Nonlinear Financial Dynamics from an Engineer's Point of View
}

\author{
C. K. Volos ${ }^{1}$, I. M. Kyprianidis ${ }^{2}$, S. G. Stavrinides ${ }^{2}$, I. N. Stouboulos ${ }^{2}$, \\ L. Magafas ${ }^{3}$ and A. N. Anagnostopoulos ${ }^{2}$ \\ ${ }^{1}$ Faculty of Mathematics and Engineering Studies, University of Military Education, 16673, Greece \\ ${ }^{2}$ Physics Department, Aristotle University of Thessaloniki, Thessaloniki, 54124, Greece \\ ${ }^{3}$ Dep. of Electrical Engineering, Technological and Educational Institute of Kavala, Greece.
}

\begin{abstract}
In this report the dynamic behaviour of a nonlinear finance chaotic system was modeled by an electronic circuit and its behaviour was studied by means of electronic circuit simulation tools. A recently new proposed finance system is used. For this reason, a totally different approach in the study of its dynamic behaviour by using the bifurcation diagram is followed, in regard to previous work. The numerical tools of this approach were not only the bifurcation diagram, but also the maximum Lyapunov exponents and the phase portraits. Various phenomena concerning the chaos theory, such as the route to chaos through the mechanism of period doubling, internal and boundary crisis and the coexistence of attractors, were observed.
\end{abstract}

Keywords: Nonlinear financial system, chaos, nonlinear circuit, bifurcation, Lyapunov exponents.

\section{Introduction}

Research activities in various fields of natural sciences, confirm the fact that nonlinear systems, exhibiting chaotic behaviour, have triggered investigation on related phenomena [1-4]. This is due both to rich dynamics and sensitivity on initial conditions that nonlinear systems demonstrate. Chaotic phenomena have also been observed in Economics by the middle of 80 s. Since then and especially the last few years a scientific field namely Econophysics, provides an alternative approach, aiming to study the particularly complex dynamics of real economic systems. In this direction, researchers are trying to explain the main features of economic theory such as structural changes, irregular (erratic) micro- and macro-economic fluctuations.

In order to fix an economic model, economists take into consideration, in a first step, only endogenous variables. This way the behaviour of the economic model is simplified. The absence of external excitation in such a model is analogous to an autonomous electronic circuit. Then the economic model is enriched with exogenous variables, describing forces not directly related to the economic model, such as political events, physical disasters etc. The introduction of external excitation is analogous to a non-autonomous electronic circuit. Therefore, complexity of these models makes accurate economic forecasting very difficult.

In many fields of economics such as funding, stocks and social economics, the diversity and complexity are unfolded in the internal structure of models that interact with external drives, due to the interaction of nonlinear factors (economic or social) with all kinds of economic problems. This was the main reason for the introduction of nonlinear dynamics to the study of economic models. As examples of nonlinear

\footnotetext{
*E-mail address: stavros@physics.auth.gr

ISSN: 1791-2377 @ 2011 Kavala Institute of Technology. All rights reserved.
}

systems used in economic models, are the well-known van der Pol model [5, 6] and others [7-11].

It is known, that nonlinear electronic circuits have been used extensively for modeling and simulating the dynamic behaviour of interdisciplinary nonlinear systems [12-15]. The aim of this work is to examine the dynamic behaviour of a finance model from an electronics engineer point of view. So, for this reason, a recently newly proposed finance system which demonstrates chaotic behaviour, for specific values of its parameters [16], is used. An analog electronic circuit emulating its behaviour is proposed and its rich dynamic behaviour is studied by means of electronic circuit simulation tools, such as PSpice.

\section{The Finance Chaotic Model}

Recently, a third-order dynamical model, describing a finance system, was reported [10, 11]. The model describes the time variations of three state variables, namely the interest rate $\mathrm{X}$, the investment demand $\mathrm{Y}$, and the price index $\mathrm{Z}$. This nonlinear finance chaotic system is described by the following set of differential equations:

$$
\left\{\begin{array}{l}
\frac{d X}{d t}=Z+(Y-\alpha) X \\
\frac{d Y}{d t}=1-b Y-X^{2} \\
\frac{d Z}{d t}=-X-c Z
\end{array}\right.
$$

Parameters $\alpha, b$ and $\mathrm{c}$ stand for: the saving amount, the cost per-investment ratio and the elasticity of demand of 
commercial markets, respectively. All three parameters possess a positive value $(\alpha \geq 0, \quad \mathrm{~b} \geq 0, \mathrm{c} \geq 0)$.

Under the linear transformation $\mathrm{x}(\mathrm{t})=\mathrm{X}(\mathrm{t})$, $\mathrm{y}(\mathrm{t})=\mathrm{Y}(\mathrm{t})-1 / \mathrm{b}, \mathrm{z}(\mathrm{t})=\mathrm{Z}(\mathrm{t})$, equation system $(1)$ turns into equation system (2) [16].

$$
\left\{\begin{array}{l}
\frac{d x}{d t}=e x+z+x y \\
\frac{d y}{d t}=-b y-x^{2} \\
\frac{d z}{d t}=-x-c z
\end{array}\right.
$$

Parameter e, in this work, is defined as follows:

$$
\mathrm{e}=\frac{1}{\mathrm{~b}-\mathrm{a}}
$$

This is a mere difference between the equation system (2) and the corresponding equation system presented in [16].

Also, in [16] the long-term dynamical behaviour of the finance chaotic system (2) was studied mainly by dividing the system into two subsystems. Here, a more general approach of the dynamic behaviour, based on the analysis of the produced bifurcation diagram of the specific system (2) is followed, in order to model the system with an electronic circuit by using well-known electronic circuit simulation tools. Therefore, a dynamical analysis of the system (2), such as symmetry and equilibrium points, is followed. Also, in the next section the nonlinear circuit which modeled the finance chaotic system is studied.

Regarding the dynamic behaviour of the nonlinear finance system described in Eq. (2), the following should be highlighted: This system remains invariant under the transformation $(\mathrm{x}, \mathrm{y}, \mathrm{z}) \rightarrow(-\mathrm{x}, \mathrm{y},-\mathrm{z})$, for any arbitrary parameters $\mathrm{e}, \mathrm{b}, \mathrm{c}$, indicating that the finance chaotic system (2) is symmetric with respect to the y-axis, that depicts normalized investment demand.

According to the value of the expression $(\mathrm{ec}-1)$, the resulting system (2) possesses the following equilibrium points:

- If ec $-1 \leq 0$, the system has only one equilibrium point: $\mathrm{S}_{1}(0,0,0)$.

- If ec $-1>0$, the system has three equilibrium points:

$\mathrm{S}_{1}(0, \quad 0, \quad 0), \quad \mathrm{S}_{2}\left(-\sqrt{\frac{(\mathrm{ec}-1) \mathrm{b}}{\mathrm{c}}},-\frac{\mathrm{ec}-1}{\mathrm{c}}, \frac{1}{\mathrm{c}} \sqrt{\frac{(\mathrm{ec}-1) \mathrm{b}}{\mathrm{c}}}\right)$,

$\mathrm{S}_{3}\left(\sqrt{\frac{(\mathrm{ec}-1) \mathrm{b}}{\mathrm{c}}},-\frac{\mathrm{ec}-1}{\mathrm{c}},-\frac{1}{\mathrm{c}} \sqrt{\frac{(\mathrm{ec}-1) \mathrm{b}}{\mathrm{c}}}\right)$

\section{The Proposed Circuit}

The circuit topology that was adopted in order to realize the equation set (2), is presented in Fig 1 . Two multipliers $U_{1}$, $\mathrm{U}_{2}\left(\right.$ AD633JN) and four identical operational amplifiers $\mathrm{U}_{3}$, $\mathrm{U}_{4}, \mathrm{U}_{5}, \mathrm{U}_{6}$ (LF411), were used. In the equation set (2), parameters e, $\mathrm{b}$ and $\mathrm{c}$ were defined as follows:

$\mathrm{e}=\frac{\mathrm{R}_{2}}{\mathrm{R}_{1}}, \mathrm{~b}=\frac{\mathrm{R}_{2}}{\mathrm{R}_{3}}, \mathrm{c}=\frac{\mathrm{R}_{2}}{\mathrm{R}_{\mathrm{X}}}$
The circuit element values were: $R_{1}=1 \mathrm{k} \Omega, R_{2}=10 \mathrm{k} \Omega$, $\mathrm{R}_{3}=100 \mathrm{k} \Omega$ and $\mathrm{C}=10 \mathrm{nF}$, while $\mathrm{R}_{\mathrm{X}}$ was varied. Consequently, the parameter values were $\mathrm{e}=10, \mathrm{~b}=0.1$, while c served as the control parameter.

Finally, the power supplies for all the ICs used were $\pm 15 \mathrm{~V}$. It should be mentioned that the signals $\mathrm{x}, \mathrm{y}$, and $\mathrm{z}$ represent the voltages at the outputs of the operational amplifiers $\mathrm{U}_{3}, \mathrm{U}_{4}$ and $\mathrm{U}_{5}$, respectively (Fig 1).

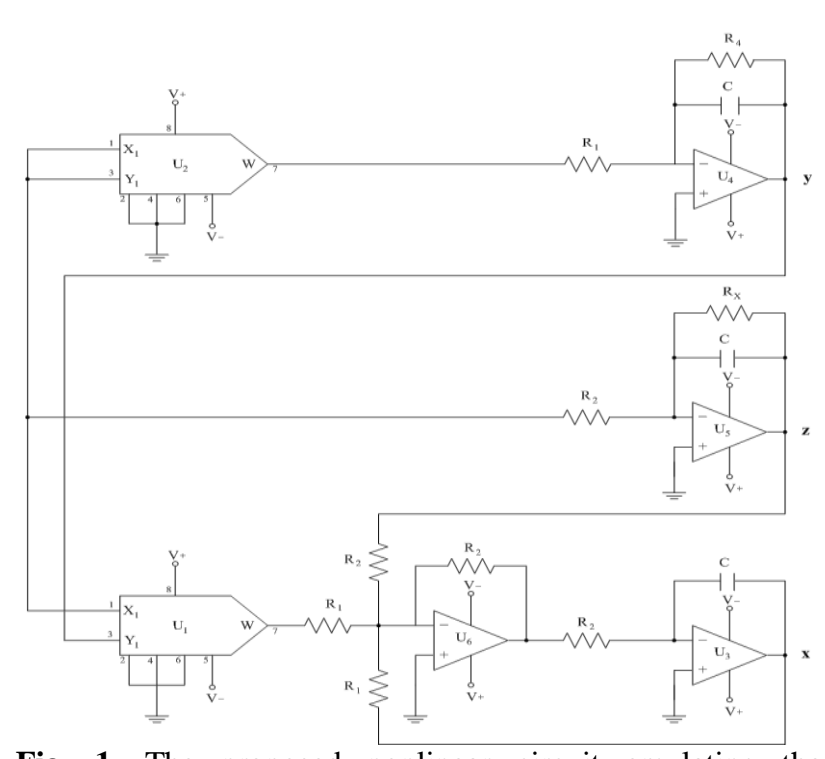

Fig. 1. The proposed nonlinear circuit emulating the nonlinear finance system

\section{Simulation Results}

In order to study and illustrate the global dynamic behaviour of the proposed nonlinear electronic circuit of Fig 1, and consequently the dynamics of the corresponding finance system, numerical simulation of system (2) was used, by employing a fourth-order Runge - Kutta algorithm.

In Fig 2 the bifurcation diagram of $y$ versus $c$ (bifurcation parameter) and the corresponding diagram of maximum Lyapunov exponent versus c, are presented, depicting the periodic and chaotic regimes, for the finance system (2). These diagrams were numerically produced by decreasing parameter $\mathrm{c}$, beginning from $\mathrm{c}=1.500$ to $\mathrm{c}=0.500$ with a step $\Delta \mathrm{c}=0.001$ and a time step $\Delta \mathrm{t}=0.001$, using the Poincare section at $\mathrm{x}=0$. Initial conditions were set to $\left(\mathrm{x}_{0}, \mathrm{y}_{0}, \mathrm{z}_{0}\right)=(3,2,1)$. The maximum Lyapunov exponent diagram was calculated by employing the Wolf $e t$ al method on the time-series of $\mathrm{y}(\mathrm{t})$. According to the numerical analysis, when the maximum Lyapunov exponent is positive, the system (circuit) is considered to operate in a chaotic mode, while when the maximum Lyapunov exponent is zero the system (circuit) turns to a periodic state.

By choosing certain values of parameter $c$ from various regions of the bifurcation diagram, the proper value of the involved resistor $R_{X}$ was determined. Then the behaviour of the nonlinear circuit was further studied by employing the electronic circuit simulation program PSpice. In detail, phenomena of the finance system, related to the nonlinear dynamic theory, are discerned in the bifurcation diagram of Fig 2. 


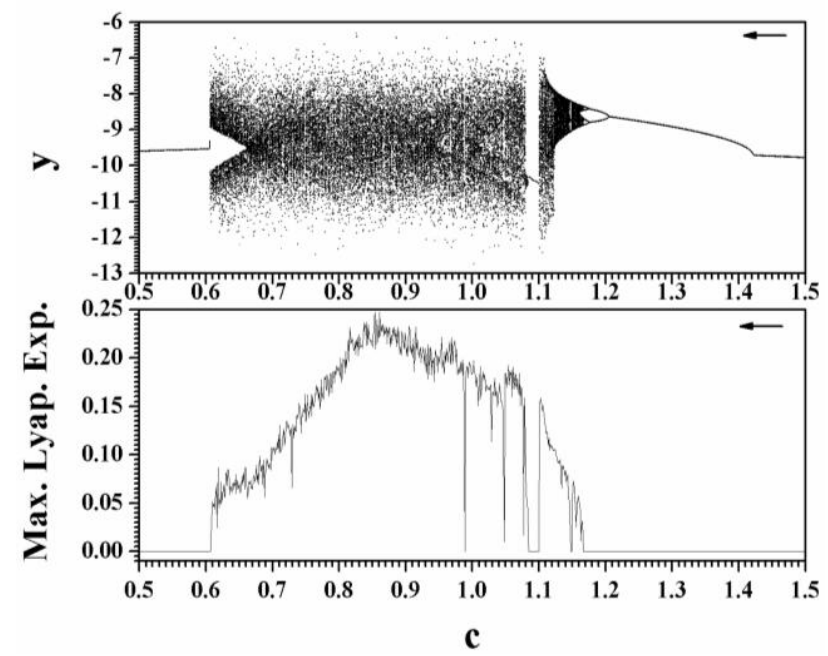

Fig. 2. Bifurcation diagram of $\mathrm{y}$ versus $\mathrm{c}$, for $\mathrm{e}=10, \mathrm{~b}=0.1$.

The first observed phenomenon is the well-known period doubling route to chaos. As the parameter $\mathrm{c}$ is decreased starting from $\mathrm{c}=1.500$, the system remains always in a period-1 steady state (Fig 3a) until $\mathrm{c}=1.205$. Then the circuit period doubles $(1.205 \geq \mathrm{c}>1.175)$, as it is shown in Fig 3(b). In the region $1.175 \geq \mathrm{c}>1.168$ (Fig 3(c)) it doubles its period again (period-4). Finally the circuit operation, enters a chaotic mode, as shown in Fig 3(d).

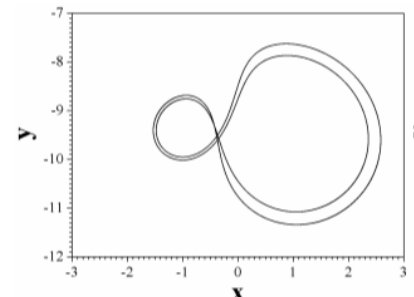

(a)

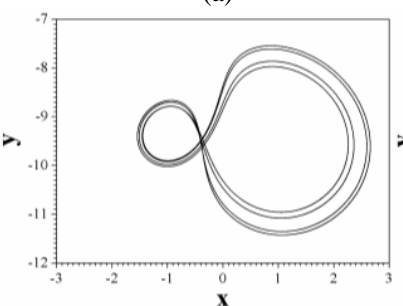

(c)

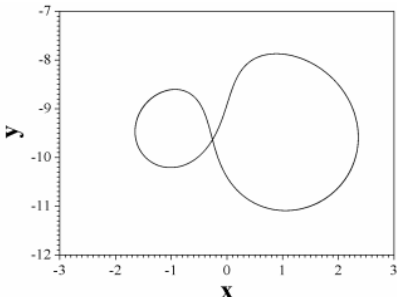

(b)

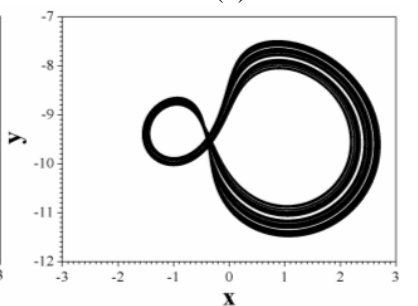

(d)
Fig. 3. Phase portraits showing the route to chaos through the mechanism of period doubling, for $\mathrm{e}=10, \mathrm{~b}=0.1$ and (a) $\mathrm{c}=1.300$ (period-1 state), $(\mathrm{b}) \mathrm{c}=1.190$ (period-2 state), (c) $\mathrm{c}=1.170 \quad$ (period-4 state) and (d) $c=1.158$ (chaotic state).

For $\mathrm{c}<1.123$, the system's attractor remains chaotic, but now comprises a much wider area that contains the smallest previous one. This change is accomplished by undergoing through a phenomenon known as internal crisis [18]. In Fig 4 the expanded chaotic attractor for $\mathrm{c}=1\left(\mathrm{R}_{\mathrm{X}}\right.$ is adjusted to $10 \mathrm{k} \Omega$ ), is shown. The 3D attractor of Fig 4(a) looks like a "fluttering butterfly", and it is clear that the dynamics of the system, in this chaotic region, are quite more complex. In Fig 4(b)-4(d), three phase portrait projections in 2Ds, are shown; while in Fig 5 the corresponding time-series of signals $\mathrm{x}, \mathrm{y}$, and $\mathrm{z}$, further confirmed the circuit chaotic behaviour and pro rata of the finance system (2) (for e $=10$, $\mathrm{b}=0.1$ and $\mathrm{c}=1)$.

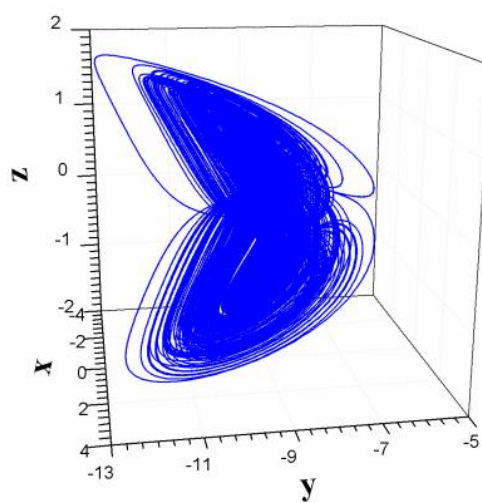

(a)

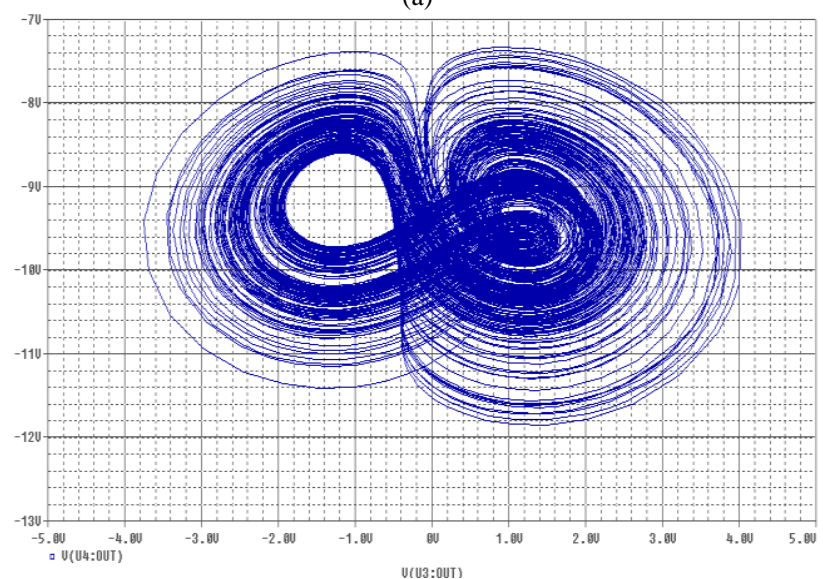

(b)

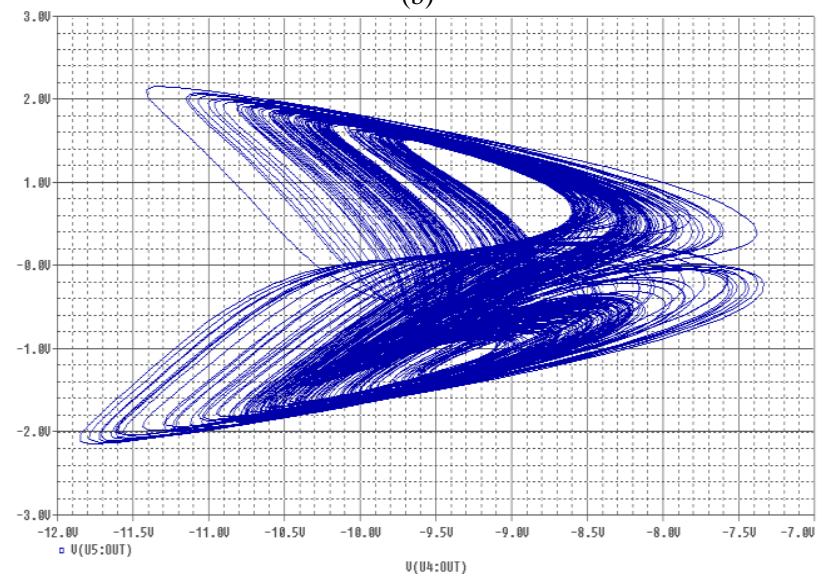

(c)

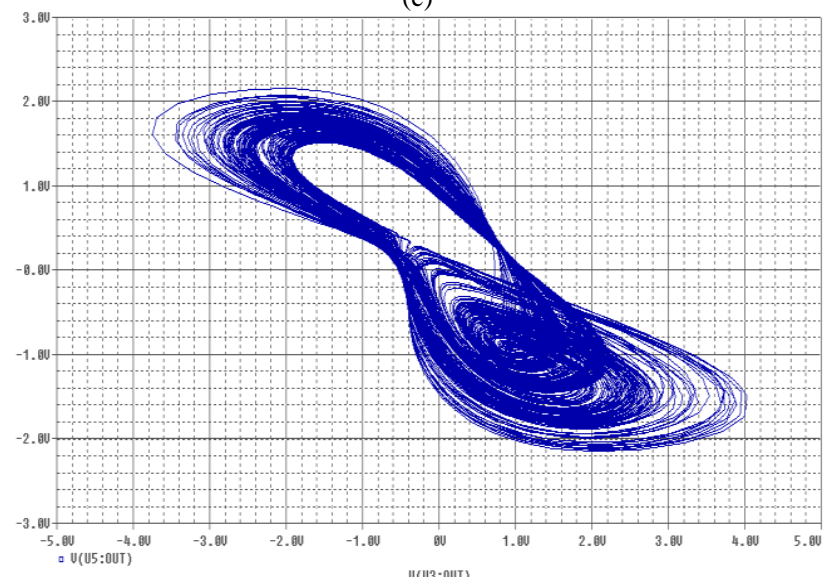

(d)

Fig. 4. (a) 3D attractor (b) y vs $x$, (c) $z$ vs $x$ and (d) $z$ vs $y$, for e $=10, b$ $=0.1$ and $\mathrm{c}=1$ 


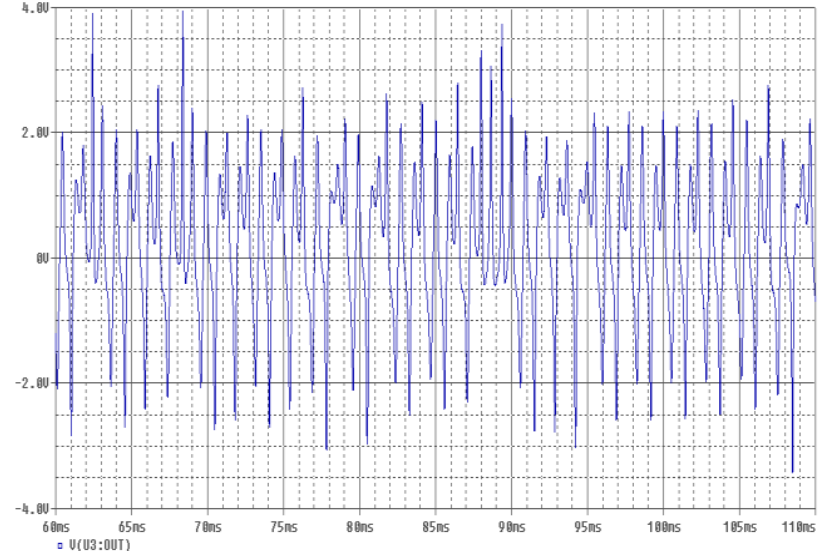

(a)

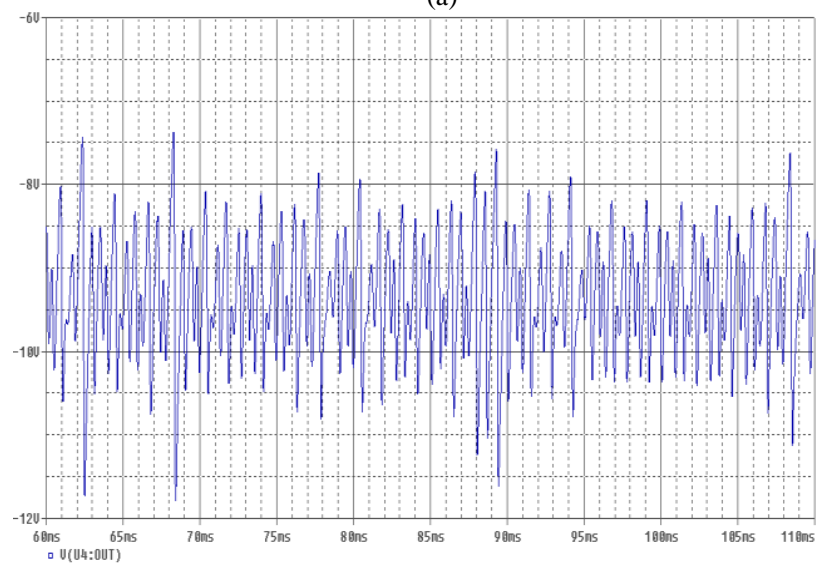

(b)

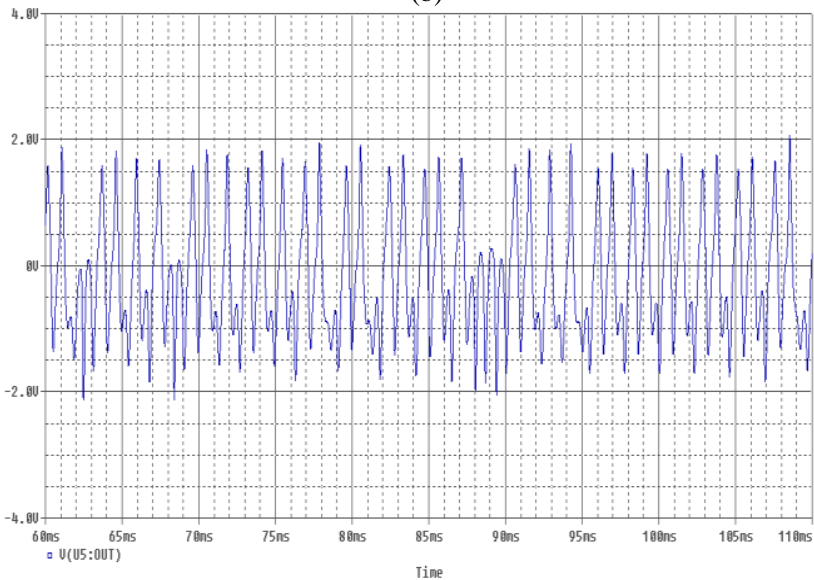

(c)

Fig. 5. The chaotic time series for $\mathrm{e}=10, \mathrm{~b}=0.1$ and $\mathrm{c}=1$, of $(\mathrm{a}): \mathrm{x}(\mathrm{t})$, (b): $y(t)$ and $(c): z(t)$

For $\mathrm{c}=0.606$ an interesting phenomenon is observed in the bifurcation diagram (Fig 2), namely boundary crisis. The system undergoes an almost abrupt transition from chaos to a periodic state (period-1). The corresponding circuit phase portraits of this periodic state, for $\mathrm{c}=0.6$, just below the critical point, are shown in Fig 6.

Finally, the phenomenon of coexistence of attractors is investigated. In Fig 7 the phase portraits for $e=10, b=0.1$ and $\mathrm{c}=1.4$, which were produced for two sets of initials conditions with opposite signs for $\mathrm{x}$ and $\mathrm{z}$, are shown. Two symmetrical, with respect to the $y$-axis, period-1 limit cycles, appear in this Figure. This is a consequence of the characteristic that the system remains invariant under the transformation $(\mathrm{x}, \mathrm{y}, \mathrm{z}) \rightarrow(-\mathrm{x}, \mathrm{y},-\mathrm{z})$, as indicated.

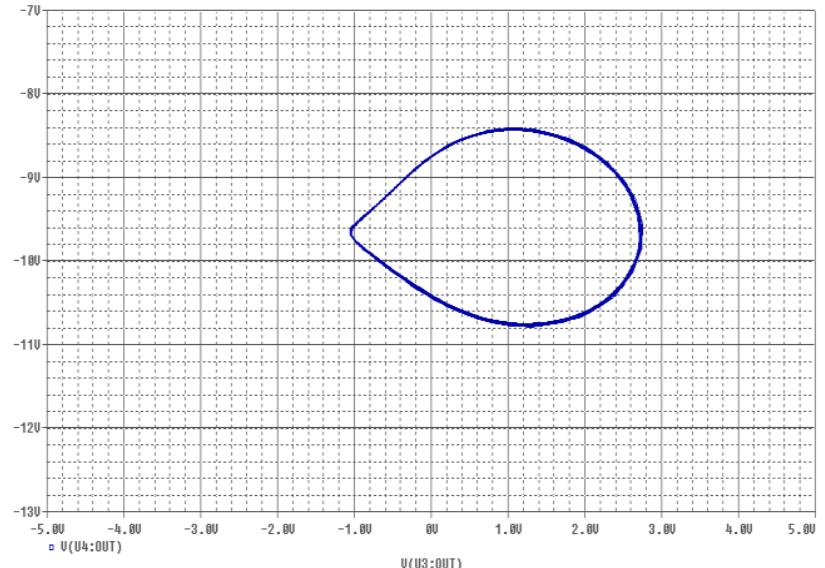

(a)

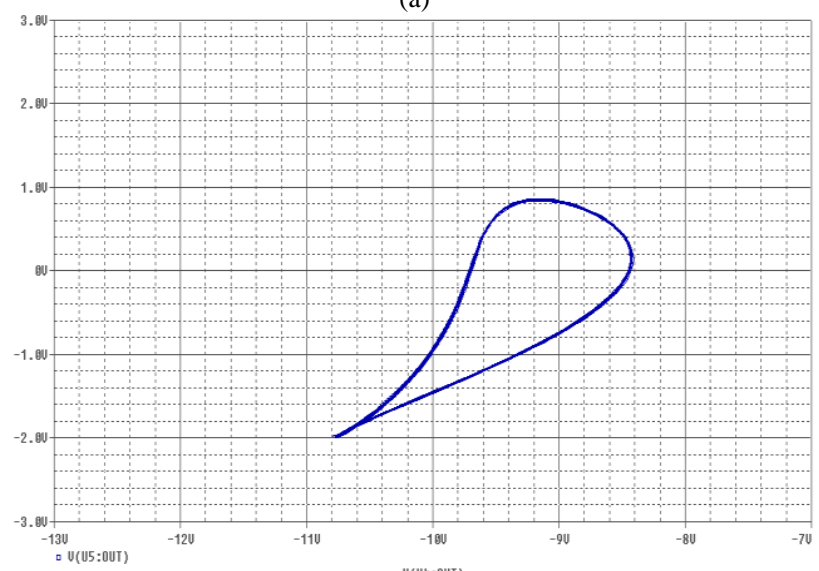

(b)

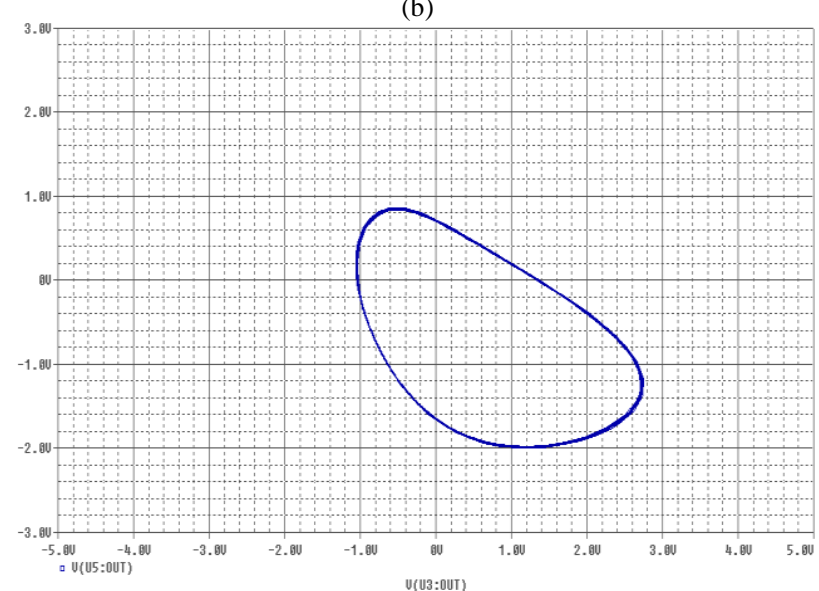

(c)

Fig. 6. Phase portraits of the system, which was produced by PSpice in: (a) y vs x plane, (b) z vs x plane and (c) $\mathrm{z}$ vs y plane, for e $=10, \mathrm{~b}=0.1$ and $\mathrm{c}=0.6$.

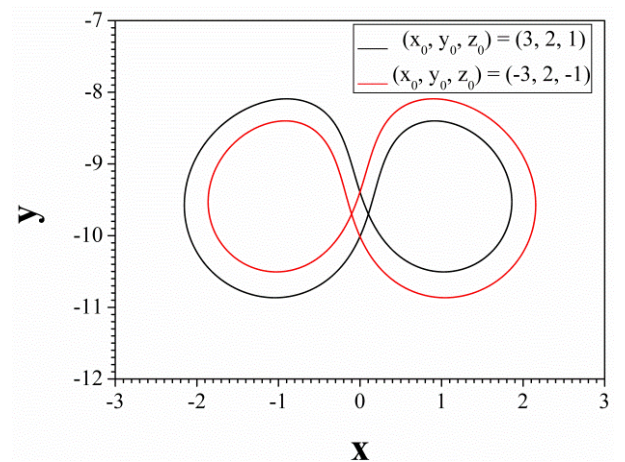

Fig. 7. Phase portraits in $y$ vs $x$ plane, for $\mathrm{e}=10, \mathrm{~b}=0.1$ and $\mathrm{c}=1.4$ with two different sets of initial conditions, $\left(\mathrm{x}_{0}, \mathrm{y}_{0}, \mathrm{z}_{0}\right)=(3,2,1)$ with black line and for $\left(\mathrm{x}_{0}, \mathrm{y}_{0}, \mathrm{z}_{0}\right)=(-3,2,-1)$ with red line. 


\section{Conclusions}

In this paper, the dynamics of a nonlinear system, describing the behaviour of a finance model, were investigated. The system is a newly proposed one and it demonstrates chaotic behaviour for specific values of its parameters. The global dynamical behaviour of the system was observed by using the bifurcation diagram, in order to find out the regions of chaotic or periodic behaviour. The study of the system's behaviour was carried out both by numerically solving the differential equations (2) and by applying PSpice simulation on a circuit. We propose here that this new system emulates a specific finance system behaviour. In this circuit two multipliers were used, to implement the nonlinear terms of the system.

Both numerical solutions as well as electronic circuit simulation, revealed the rich chaotic dynamics of this finance system. Very interesting phenomena were observed. The well-known route to chaos through period doubling is the first one. Internal and boundary crisis phenomena were also observed. Finally, the coexistence of attractors, which is a consequence of the endogenous characteristic that the system remains invariant under the transformation $(\mathrm{x}, \mathrm{y}, \mathrm{z}) \rightarrow(-\mathrm{x}, \mathrm{y},-\mathrm{z})$, was confirmed.

\section{References}

1. G. Chen and X. Dong, From Chaos to Order: Methodologies, Perspectives and Applications, World Scientific, Singapore, (1998).

2. I. R. Epstein and J. A. Pojman, An Introduction to Nonlinear Chemical Dynamics: Oscillations, Waves, Patterns, and Chaos (Topics in Physical Chemistry), Oxford University Press, (1998).

3. J. Walleczek, Self-Organized Biological Dynamics and Nonlinear Control: Toward Understanding Complexity, Chaos and Emergent Function in Living Systems, Cambridge University Press, (2006).

4. S. H. Strogatz, Nonlinear Dynamics and Chaos: With Applications to Physics, Biology, Chemistry, and Engineering, Westview Press, (2001).

5. L. Pribylová, Bifurcation Routes to Chaos in an Extended van der Pol's Equation Applied to Economic Models, Electronic Journal of Differential Equations, 2009 (53) 1 (2009).

6. A. L. Chian, F. A. Borotto, E. L. Rempel and C. Rogers, Attractor Merging Crisis in Chaotic Business Cycles, Chaos, Solitons \& Fractals 24869 (2005).

7. L. D. Cesare, M. Sportelli, A dynamic IS-LM Model with Delayed Taxation Revenues, Chaos, Solitons \& Fractals 25233 (2005).

8. H. W. Lorenz and H. E. Nusse, Chaotic Attractors, Chaotic Saddles, and Fractal Basin Boundaries: Goodwin's Nonlinear Accelerator Model Reconsidered, Chaos, Solitons \& Fractals, 13 957 (2002).

9. J. H. Ma and Y. S. Chen, Study for the Bifurcation Topological Structure and the Global Complicated Character of a Kind of Nonlinear Finance System (I), Appl. Math. Mech. (English ed.), 22 1240 (2001).

10. J. H. Ma and Y. S. Chen, Study for the Bifurcation Topological Structure and the Global Complicated Character of a Kind of
Nonlinear Finance System (II), Appl. Math. Mech. (English ed.), 221375 (2001)

11. W. C. Chen, Nonlinear Dynamics and Chaos in a Fractional-Order Financial System, Chaos, Solitons \& Fractals, 361305 (2008).

12. Ch. K. Volos, I. M. Kyprianidis, I. N. Stouboulos and A. N. Anagnostopoulos, Experimental Study of the Dynamic Behaviour of a Double Scroll Circuit, J. Applied Functional Analysis, 4703 (2009).

13. I. M. Kyprianidis, Ch. K. Volos, S. G. Stavrinides, I. N. Stouboulos and A. N. Anagnostopoulos, Master - Slave Double Scroll Circuit Incomplete Synchronization, J. Engineering Science \& Technology Review, 341 (2010).

14. Ch. K. Volos, I. M. Kyprianidis and I. N. Stouboulos, Experimental Study of a Nonlinear Circuit Described by Duffing's Equation, In Proc. Chaos \& Complex Systems I (CCS 2006), 445 (2006).

15. Ch. K. Volos, I. M. Kyprianidis and I. N. Stouboulos, Chaotic Dynamics from a Nonlinear Circuit Based on Memristor with a Cubic Nonlinearity, In Proc. 7th Int. Conference of the Balkan Physical Union, (BPU 2009), AIP Conference Proceedings, 1203 626 (2009).

16. G. Cai and J. Huang, A New Finance Chaotic Attractor, Int. J. Nonlinear Science, 3 (3) 213 (2007).

17. A. Wolf, J. Swift, H. Swinney, J. Vastano, Determining Lyapunov Exponents from a Time Series, Physica D, 16285 (1985).

18. E. Ott, Chaos in Dynamical Systems, Cambridge University Press, (1992). 